\title{
HYPERBOLICITY OF NODAL HYPERSURFACES
}

\author{
Fedor Bogomolov*, Bruno De Oliveira** \\ * Courant Institute for Mathematical Sciences, ${ }^{* *}$ University of Miami
}

\begin{abstract}
We show that a nodal hypersurface $X$ in $\mathbb{P}^{3}$ of degree $d$ with a sufficiently large number $l$ of nodes, $l>\frac{8}{3}\left(d^{2}-\frac{5}{2} d\right)$, is algebraically quasi-hyperbolic, i.e. $X$ can only have finitely many rational and elliptic curves. Our results use the theory of symmetric differentials and algebraic foliations and give a very striking example of the jumping of the number of symmetric differentials in families.
\end{abstract}

\section{InTRODUCTION}

A compact analytic variety $X$ is said to be Kobayashi hyperbolic (Brody) if it does not contain any entire curves, i.e. there are no nonconstant holomorphic mappings $f: \mathbb{C} \rightarrow X$.

Conjecture. (Kobayashi) The general hypersurface $X$ of $\mathbb{P}^{3}$ of degree $\geq 5$ is Kobayashi hyperbolic.

We are more interested in an algebraic version of this conjecture (see below) but we recall that the results of McQuillan [Mc98] $(d \geq 36)$ and Demailly and El Goul [DeEl00] $(d \geq 21)$ which state that a very general hypersurface of degree $d \geq 21$ in $\mathbb{P}^{3}$ is Kobayashi hyperbolic (a condition holds for a very generic hypersurface of degree $d$ if it holds for all hypersurfaces not belonging to some countable union of subvarieties of the parameter space of hypersurfaces of degree $d$ ). See also [SiYe96] and [EG03] for the related problem of the hyperbolicity of the complement of an hypersurface in $\mathbb{P}^{2}$.

The algebraic version of the Kobayashi's conjecture states that the general hypersurface $X$ of $\mathbb{P}^{3}$ of degree $\geq 5$ does not contain any rational or elliptic curves, i.e. $X$ is algebraically hyperbolic. The algebraic version is, a priori, weaker but Green and Griffiths [GrGr80] formulated a conjecture which reduces the Kobayashi's hyperbolicity of $X$ to the algebraic hyperbolicity of $X$. More generally and in a broader direction,

\footnotetext{
* Partially supported by the NSF grant DMS-0100837. ** Partially supported by the NSF grant DMS-0306487
}

Typeset by $\mathcal{A M}_{\mathcal{M}} \mathcal{T}_{\mathrm{E}} \mathrm{X}$ 
the first author and separately Green and Griffiths formulated that a surface $X$ of general type contains only finitely many rational and elliptic curves, i.e. $X$ is algebraically quasi-hyperbolic (this conjecture was first explicitly written by Lang [La86]). If $X$ is a variety of general type then there is a jet-bundle $J_{k}(X)$ over $X$ with a proper subvariety $S \subset J_{k}(X)$ so that the curves in $X$ are divided into the class of regular curves and the class of special curves. The class of regular curves $C \subset X$ consists of the ones whose lifting $j_{k}\left(C^{\prime}\right)$ into $J_{k}(X)$ is not contained in $S\left(C^{\prime}\right.$ denotes the normalization of $C$ ). The class of the special curves consists of the ones whose lifting $j_{k}\left(C^{\prime}\right) \subset S$. The special curves satisfy differential equations of order $k$ defined by $S$. Now for regular curves we have a linear estimate on genus viz degree with respect to $K_{X}$. Namely $g\left(C^{\prime}\right) \geq c(X) K_{X} . C, c(X)$ is a constant depending on $X$. For special curves there isn't such estimate. But if we consider special curves of $X$ lifting a surface component of $S$ then we have boundedness results for curves of any fixed genus coming from the theory of foliations [Jo78].

On the algebraic version of the Kobayashi's conjecture, there are the Clemens's [Cl86] and Xu's [Xu94] results giving that a very general hypersurface in $\mathbb{P}^{3}$ of degree $\geq 5$ does not contain rational or elliptic curves. For higher dimensions, Ein [Ei88] (see also [Vo96]) showed that a very general hypersurface on $\mathbb{P}^{n+1}$ of degree $\geq 2 n+2$ does not contain any submanifold that is not of general type.

In this paper, we analyze how the number of nodes in a hypersurface $X$ of $\mathbb{P}^{3}$ influences the presence of symmetric differentials on the minimal resolution $Y$ of $X$ (a symmetric differential on $Y$ is a global section of $S^{m} \Omega_{Y}^{1}$ ). The existence of symmetric differentials on a surface $Y$ imposes constraints on the genus of curves on $Y$. Moreover, if a surface of general type $Y$ satisfies $h^{0}\left(Y, S^{m} \Omega_{Y}^{1}\right) \uparrow m^{3}$ then $Y$ must be algebraically quasi-hyperbolic (see section 3 of [Bo77] and [De79]). We remark that this approach to algebraic hyperbolicity has the advantage that it can also be used as an ingredient to obtain analytic hyperbolicity.

The pivotal result in this paper is that the minimal resolution $Y$ of a nodal hypersurface $X$ in $\mathbb{P}^{3}$ of degree $d$ with $l>\frac{8}{3}\left(d^{2}-\frac{5}{2} d\right)$ nodes satisfies $h^{0}\left(Y, S^{m} \Omega_{Y}^{1}\right) \uparrow m^{3}$. Hence, $Y$ and $X$ are algebraically quasi-hyperbolic. Recall, that the literature on nodal hypersurfaces gives that there are nodal hypersurfaces of degree $d$ with number of nodes $l>\frac{8}{3}\left(d^{2}-\frac{5}{2} d\right)$ for degrees $d \geq 6$ (see [Mi83]). The existence of symmetric differentials on the minimal resolutions of nodal hypersurfaces has some striking consequences. It is well known that the smooth hypersurfaces in $\mathbb{P}^{3}$ have no symmetric differentials ([Br 71], [Bo78] and [Sa79]). Our result brings the theory of symmetric differentials back as a tool to obtain (algebraic/analytical) hyperbolicity properties of hypersurfaces. Also, we get a simple example of the jumping of the number of symmetric differentials in families. Atiyah [At52] showed that a family of smooth hypersurfaces in $\mathbb{P}^{3}$ specializing to a nodal hypersurface has a simultaneous resolution. Consider the simultaneous resolution of a family whose general member is a smooth hypersurface and whose special member is a nodal hypersurface with $l>\frac{8}{3}\left(d^{2}-\frac{5}{2} d\right)$ nodes. Our results imply that the resulting family has a general member with no symmetric differentials but whose special member has plenty of symmetric differentials. We finally point out that a byproduct of this paper 
is a source of examples of special hypersurfaces that are algebraically quasi-hyperbolic. In the smooth case there are some constructions of special hyperbolic hypersurfaces in $\mathbb{P}^{3}$, see for example [ShZa00].

We would like to thank the referee for his remarks that improved the readability of this article and Jánus Kollár for his important comments.

\section{Preliminaries}

Let $E$ be a vector bundle of rank $r$ on a smooth surface $X$. The Hirzebruch-RiemannRoch theorem expresses the Euler characteristic $\chi(X, E)=\sum(-1)^{i} \operatorname{dim} H^{i}(X, \mathcal{O}(E))$ in terms of topological invariants $c_{1}(E), c_{2}(E), K=-c_{1}\left(T_{X}\right)$ and $\chi=c_{2}\left(T_{X}\right) \in H^{*}(X, \mathbb{Z})$ :

$$
\chi(X, E)=\operatorname{ch}(E) T d(X)[X]=\left(-\frac{1}{2} c_{1}(E) \cdot K+\frac{1}{2} c_{1}^{2}(E)-c_{2}(E)+\frac{r}{12}\left(K^{2}+\chi\right)\right)[X]
$$

We are interested in the asymptotic Hirzebruch-Riemann-Roch formula for $S^{m} E$ and in particular its leading term:

$$
\chi\left(X, S^{m} E\right)=\frac{m^{r+1}}{3 !}\left(c_{1}^{2}(E)-c_{2}(E)\right)+O\left(m^{r}\right)
$$

which depends on the bundle invariants and does not depend on the surface's Chern classes. We are interested in the symmetric products of the sheaf of differentials $\Omega_{X}^{1}$. So, actually in this case, the invariants of the bundle $\Omega_{X}^{1}$ are invariants of the surface $X$.

Our results require the analysis of the case where the smooth surface $Y$ is the minimal resolution of a surface $X$ with quotient (nodal) singularities. In particular, a key factor in our study is the contribution of the singularities of $X$ to the Euler characteristic $\chi\left(Y, S^{m} \Omega_{Y}^{1}\right)$. We used the general framework developed in the work of Wahl [Wa93] and Blache [B196]. In their work, they considered a local Riemann-Roch with local Chern classes [Wa 93], [B196] and the connection between the local and the global RiemannRoch [B196].

Let $(X, x)$ be the germ of a normal surface singularity and $(Y, E)$ be a good resolution of $(X, x)$, i.e the exceptional divisor $E$ over $x$ consists of smooth curves intersecting transversely. Wahl defined local Chern classes, $c_{i}(\tilde{\mathcal{F}})_{\text {loc }}$, of locally free sheaves $\tilde{\mathcal{F}}$ of rank 2 on $Y$ (Wahl also calls them, relative Chern classes since they can be viewed as classes in $\left.H^{2 i}(Y, \partial Y, \mathbb{Q})\right)$. Let $\tilde{\mathcal{F}}$ be a locally free sheaf of rank 2 then the Chern numbers are given by:

a) $c_{1}^{2}(\tilde{\mathcal{F}})_{\text {loc }}=[\operatorname{det} \tilde{\mathcal{F}}]^{2} \in \mathbb{Q}$ 
where $[\operatorname{det} \tilde{\mathcal{F}}]$ is the unique divisor $[\operatorname{det} \tilde{\mathcal{F}}]=\sum a_{i} E_{i}, E_{i}$ are the irreducible components of $E$, satisfying $\left(\sum a_{i} E_{i}\right) E_{j}=\operatorname{deg}_{E_{j}} \operatorname{det} \tilde{\mathcal{F}}$ for all $j$.

b) $c_{2}(\tilde{\mathcal{F}})_{\operatorname{loc}}=\inf \left\{\frac{1}{\operatorname{deg} f}[\mathcal{L}] \cdot[\mathcal{M}]\right\}$

where the infimum is taken over all quadruples $[W, f:(W, F) \rightarrow(Y, E), \mathcal{L}, \mathcal{M}]$ such that $f$ is a proper surjective generically finite map with $f^{-1}(E)=F$ and $\mathcal{L}, \mathcal{M}$ are line bundles fitting an exact sequence $0 \rightarrow \mathcal{L} \rightarrow f^{*} \tilde{\mathcal{F}} \rightarrow \mathcal{M} \rightarrow 0$.

In [Wa93] it was defined a modified Euler characteristic of a locally free sheaf $\tilde{\mathcal{F}}$ on $Y$ :

$$
\chi^{\prime}(\tilde{\mathcal{F}})_{x}=\operatorname{dim}\left[H^{0}(Y \backslash E, \tilde{\mathcal{F}}) / H^{0}(Y \tilde{\mathcal{F}})\right]+h^{1}(Y, \tilde{\mathcal{F}})
$$

Wahl conjectured that for a rank 2 bundle $\tilde{\mathcal{F}}$ the following asymptotic local RiemannRoch formula holds:

$$
\chi^{\prime}\left(S^{m} \tilde{\mathcal{F}}\right)_{x}=-\frac{m^{3}}{6}\left(c_{1}^{2}(\tilde{\mathcal{F}})_{\text {loc }}-c_{2}(\tilde{\mathcal{F}})_{\text {loc }}\right)+O\left(m^{2}\right)
$$

using the local Chern numbers of $\tilde{\mathcal{F}}$. This conjecture holds in the case $(X, x)$ is a nodal singularity [Wa93] and more generally when $(X, x)$ is any quotient singularity [La00].

Recall that $X$ is an orbifold if all $x \in X$ have a neighborhood $U_{x} \subset X$ such that: there is an open neighborhhod of $0 \in \mathbb{C}^{n}$ with a small finite subgroup $G_{x} \subset G L_{n}(\mathbb{C})$ such that $U_{x}=V / G_{x}$. Hence an orbifold has only quotient singularities. Consider the germ $(X, x)=(Y, y) / G_{x}$ with $(Y, y)=\left(\mathbb{C}^{n}, 0\right)$, the projection $\pi:(Y, y) \rightarrow(X, x)$ is called the local smoothing covering of $X$ at $x$. A coherent sheaf $\mathcal{S}$ on an orbifold $X$ is locally $\mathrm{V}$-free if every $x \in X$ has a neighborhood $\left(U_{x}, x\right)$ with a local smoothing $\pi:(V, y) \rightarrow\left(U_{x}, x\right)$ and a free sheaf $\tilde{\mathcal{S}}$ such that $\left.\mathcal{S}\right|_{U_{x}} \simeq \pi_{*}^{G_{x}}(\tilde{\mathcal{S}})[\mathrm{B} 196]$.

The interplay between the local and global Hirzebruch-Riemann-Roch formula for orbifolds is described in [B196]. Let $X$ be a projective orbifold with isolated singularities and $\sigma: Y \rightarrow X$ a good resolution of $X$. Let $\mathcal{F}$ be a locally $\mathrm{V}$-free sheaf on $X$ and $\tilde{\mathcal{F}}$ be a coherent sheaf on $Y$ such that $\mathcal{F} \simeq\left(\sigma_{*} \tilde{\mathcal{F}}\right)^{\vee \vee}$, then:

$$
\chi(X, \mathcal{F})=\chi(Y, \tilde{\mathcal{F}})+\sum_{x \in \operatorname{Sing} X} \chi^{\prime}(\tilde{\mathcal{F}})_{x}
$$




\section{SYMmETRIC TENSORS ON NODAL HYPERSURFACES}

The goal of this section is to show that the resolutions $Y$ of nodal hypersurfaces $X \subset \mathbb{P}^{3}$ containing a sufficiently large number of nodes have symmetric differentials. This result contrasts with the, well known, fact that smooth hypersurfaces $X \subset \mathbb{P}^{3}$ have no symmetric differentials, see (2.5). Atiyah's result on simultaneous resolution of nodal surfaces [At58] states that $Y$ lies in a smooth family $\omega: X_{t} \rightarrow \Delta$ whose central fiber $X_{0}=Y$ and the other members $X_{t}$ are smooth hypersurfaces of the same degree as $X$. So as a consequence of this section, we have perhaps the most striking example of a smooth family of projective manifolds with jumps on the dimension of the spaces of symmetric differentials. In section 3, we use the existence of symmetric differentials to obtain the algebraic quasi-hyperbolicity of nodal hypersurfaces.

Let $X$ be an hypersurface of degree $d$ in $\mathbb{P}^{n}$. We first recall what is known for smooth hypersurfaces. There are two key exact sequences for determining the properties of the sheaf of differentials $\Omega_{X}^{1}$ for a smooth $X$ :

$$
\begin{gathered}
\left.0 \rightarrow \Omega_{\mathbb{P}^{n}}^{1}\right|_{X} \rightarrow \bigoplus^{n+1} \mathcal{O}_{X}(-1) \rightarrow \mathcal{O}_{X} \rightarrow 0 \\
\left.0 \rightarrow \mathcal{O}_{X}(-d) \rightarrow \Omega_{\mathbb{P}^{n}}^{1}\right|_{X} \rightarrow \Omega_{X}^{1} \rightarrow 0
\end{gathered}
$$

Our main interest is on hypersurfaces in $\mathbb{P}^{3}$. For this case, the Chern character identity for the exact sequence $(2.1)$ gives $\operatorname{ch}\left(\left.\Omega_{\mathbb{P}^{3}}^{1}\right|_{X}\right)=\operatorname{ch}\left(\bigoplus^{4} \mathcal{O}_{X}(-1)\right)=\left(1+c_{1}\left(\mathcal{O}_{X}(-1)\right)^{4}\right.$. The exact sequence $(2.2)$ gives $\operatorname{ch}\left(\Omega_{X}^{1}\right) \operatorname{ch}\left(\mathcal{O}_{X}(-d)\right)=\operatorname{ch}\left(\left.\Omega_{\mathbb{P}^{3}}^{1}\right|_{X}\right)$. Hence (noting that $\left.c_{1}\left(\mathcal{O}_{X}(1)\right) c_{1}\left(\mathcal{O}_{X}(1)\right)=d\right)$ :

$$
\begin{gathered}
c_{1}\left(\Omega_{X}^{1}\right)=(d-4) c_{1}\left(\mathcal{O}_{X}(1)\right) \\
c_{2}\left(\Omega_{X}^{1}\right)=c_{2}\left(\left.\Omega_{\mathbb{P}^{3}}^{1}\right|_{X}\right)-c_{1}\left(\Omega_{X}^{1}\right) c_{1}\left(\mathcal{O}_{X}(-d)\right)=d\left(d^{2}-4 d+6\right)
\end{gathered}
$$

The formula (1.2) then gives the asymptotic behavior of the Euler characteristic $\chi\left(X, S^{m} \Omega_{X}^{1}\right)$ on $X$ :

$$
h^{0}\left(X, S^{m} \Omega_{X}^{1}\right)-h^{1}\left(X, S^{m} \Omega_{X}^{1}\right)+h^{2}\left(X, S^{m} \Omega_{X}^{1}\right)=\frac{1}{6}\left(10 d-4 d^{2}\right) m^{3}+O\left(m^{2}\right)
$$

As is well known, if $X$ is a smooth hypersurface in $\mathbb{P}^{n}$ then it has no symmetric differentials, i.e:

$$
H^{0}\left(X, S_{5}^{m} \Omega_{X}^{1}\right)=0
$$


There are at least 3 different proofs of this result ([Br71], [Bo78] and [Sa79]). Sakai's version is the shortest to describe. Consider the spectral sequence: $E_{1}^{p q}=H^{q}\left(X, \wedge^{-p} N_{X / \mathbb{P}^{3}}^{\vee} \otimes\right.$ $\left.\left.S^{m+p} \Omega_{\mathbb{P}^{n}}^{1}\right|_{X}\right) \Longrightarrow H^{p+q}\left(X, S^{m} \Omega_{X}^{1}\right) \quad(-m \leq p \leq 0)$. The vanishing (2.5) follows from $H^{i}\left(X,\left.S^{m} \Omega_{\mathbb{P}^{n}}^{1}\right|_{X} \otimes \mathcal{O}_{X}(t)\right)=0$ for $i=0,1$ and $m \geq t+2$.

Let $X$ be a nodal hypersurface in $\mathbb{P}^{3}$, i.e. its singularities are ordinary double points. Denote by $Y$ the minimal resolution of $X$ with resolution map $\sigma: Y \rightarrow X$. It follows from Atiyah's result on simultaneous resolution of nodal surfaces [At58] that $Y$ lies in a smooth family $\omega: X_{t} \rightarrow \Delta$ whose central fiber $X_{0}=Y$ and the other members $X_{t}$ are smooth hypersurfaces of the same degree as $X$. In fact, these $X_{t}$ come, after base change, from a family of hypersurfaces whose central fiber is $X$. This implies that every coherent sheaf $\mathcal{F}$ on the family $X_{t}$ that is flat over $\Delta$ has constant Euler characteristic $\chi\left(X_{t}, \mathcal{F}_{t}\right)$ in the family. In particular, one gets:

$$
\chi\left(Y, S^{m} \Omega_{Y}^{1}\right)=\chi\left(X_{t}, S^{m} \Omega_{X_{t}}^{1}\right)=\frac{1}{6}\left(10 d-4 d^{2}\right) m^{3}+O\left(m^{2}\right)
$$

Let $X$ be a nodal hypersurface in $\mathbb{P}^{3}$ with $l$ nodes and $Y$ be the minimal resolution of $X$ with resolution map $\sigma: Y \rightarrow X$ and exceptional locus $E=\amalg_{i} E_{i}$ where the $E_{i}$ are $(-2)$-rational curves. The nodal hypersurface $X$ is an orbifold and $\Omega_{X}^{1}$, the reflexive sheaf extending the locally free sheaf $\left.\Omega_{X}^{1}\right|_{X_{\text {reg }}}$, is a locally $\mathrm{V}$-free sheaf on $X$. On the resolution $Y$ there are two natural sheaves of differentials to consider $\Omega_{Y}^{1}$ and $\Omega_{Y}^{1}(\log E)$. It is clear that $\left(\sigma_{*} \Omega_{Y}^{1}\right)^{\vee \vee}=\Omega_{X}^{1}$ and $\left(\sigma_{*} \Omega_{Y}^{1}(\log E)\right)^{\vee \vee}=\Omega_{X}^{1}$.

Recalling that the class of reflexive sheaves is not closed under tensor product operations we define $\hat{S}^{m} \Omega_{X}^{1} \equiv\left(S^{m} \Omega_{X}^{1}\right)^{\vee \vee}$ (note that $\left(\sigma_{*} S^{m} \Omega_{Y}^{1}\right)^{\vee \vee}=\left(\sigma_{*} S^{m} \Omega_{Y}^{1}(\log E)\right)^{\vee \vee}=$ $\hat{S}^{m} \Omega_{X}^{1}$ ). Formula (1.5) gives the relation between the Euler characteristics of both sheaves $S^{m} \Omega_{Y}^{1}$ and $S^{m} \Omega_{Y}^{1}(\log E)$ on $Y$ and the Euler characteristic of $\hat{S}^{m} \Omega_{X}^{1}$ on $X$.

$$
\begin{gathered}
\chi\left(X, \hat{S}^{m} \Omega_{X}^{1}\right)=\chi\left(Y, S^{m} \Omega_{Y}^{1}\right)+\sum_{x_{i} \in \operatorname{Sing} X} \chi^{\prime}\left(S^{m} \Omega_{Y}^{1}\right)_{x_{i}} \\
\chi\left(X, \hat{S}^{m} \Omega_{X}^{1}\right)=\chi\left(Y, S^{m} \Omega_{Y}^{1}(\log E)\right)+\sum_{x_{i} \in \operatorname{Sing} X} \chi^{\prime}\left(S^{m} \Omega_{Y}^{1}(\log E)\right)_{x_{i}}
\end{gathered}
$$

Lemma 2.1. Let $(Y, E)$ be a good resolution of the germ $(X, x)$ of a nodal surface singularity. Then the asymptotic local Euler characteristic for $S^{m} \Omega_{Y}^{1}$ and $S^{m} \Omega_{Y}^{1}(\log E)$ is given by the formula:

$$
\chi^{\prime}\left(S^{m} \Omega_{Y}^{1}\right)_{x}=\chi^{\prime}\left(S^{m} \Omega_{Y}^{1}(\log E)\right)_{x}=\frac{1}{4} m^{3}+O\left(m^{2}\right)
$$


Proof. To find the leading terms of $\chi^{\prime}\left(S^{m} \Omega_{Y}^{1}\right)_{x}$ and $\chi^{\prime}\left(S^{m} \Omega_{Y}^{1}(\log E)\right)_{x}$, we recall what is known about their respective local Chern numbers [Wahl93]:

$$
\begin{gathered}
c_{1}^{2}\left(\Omega_{Y}^{1}\right)_{\mathrm{loc}}=0 \quad c_{1}^{2}\left(\Omega_{Y}^{1}(\log E)\right)_{\mathrm{loc}}=\left(c_{1}\left(\Omega_{Y}^{1}\right)_{\mathrm{loc}}+E\right)^{2}=-2 \\
c_{2}\left(\Omega_{Y}^{1}(\log E)\right)_{\mathrm{loc}}=-\frac{1}{2} \quad c_{2}\left(\Omega_{Y}^{1}\right)_{\mathrm{loc}}=c_{2}\left(\Omega_{Y}^{1}(\log E)_{\mathrm{loc}}+\chi_{\mathrm{top}}(E)=\frac{3}{2}\right.
\end{gathered}
$$

The key facts, for arriving to the above numbers, are that the nodes are quotient singularities with group $\mathbb{Z}_{2}$ and that the following exact sequence holds:

$$
0 \rightarrow \Omega_{Y}^{1} \rightarrow \Omega_{Y}^{1}(\log E) \rightarrow \mathcal{O}_{E} \rightarrow 0
$$

The formula (1.4) and the local Chern numbers (2.9) provide us with the desired equality (2.8).

The modified local Euler characteristic $\chi^{\prime}\left(S^{m} \Omega_{Y}^{1}\right)_{x}$ is composed of two terms $\operatorname{dim}\left[H^{0}(Y \backslash\right.$ $\left.E, \tilde{\mathcal{F}}) / H^{0}(Y \tilde{\mathcal{F}})\right]$ and $h^{1}(Y, \tilde{\mathcal{F}})$, see formula $(1.3)$. The following calculation clarifies each of the two terms for a nodal singularity.

Lemma 2.2. Let $(Y, E)$ be a good resolution of the germ $(X, x)$ of a nodal surface singularity. Then:

$$
\operatorname{dim}\left[H^{0}\left(Y \backslash E, S^{m} \Omega_{Y}^{1}\right) / H^{0}\left(Y, S^{m} \Omega_{Y}^{1}\right)\right]=\frac{1}{4} m^{3}+O\left(m^{2}\right)
$$

and:

$$
h^{1}\left(Y, S^{m} \Omega_{Y}^{1}\right)<C m^{2}
$$

Proof. Let $\sigma:(Y, E) \rightarrow(X, x)$ be the minimal resolution of the nodal singularity. The germ of a nodal singularity $(X, x)$ has a local smoothing covering $\pi:\left(X^{\prime}, x^{\prime}\right) \rightarrow(X, x)$ with group $\mathbb{Z}_{2}$ (i.e. $(X, x)$ is the quotient of a smooth germ $\left(X^{\prime}, x^{\prime}\right)$ by $\mathbb{Z}_{2}$ with fixed point set $\left.x^{\prime}\right)$. Denote by $f:\left(Y^{\prime}, E^{\prime}\right) \rightarrow\left(X^{\prime}, x^{\prime}\right)$ the blow up of $\left(X^{\prime}, x^{\prime}\right)$ at $x^{\prime}$ and $g:\left(Y^{\prime}, E^{\prime}\right) \rightarrow(Y, E)$ the double covering ramified at $E$ making $\pi \circ f=\sigma \circ g:\left(Y^{\prime}, E^{\prime}\right) \rightarrow$ $(X, x)$. There are natural bijections between $H^{0}\left(Y \backslash E, S^{m} \Omega_{Y}^{1}\right), H^{0}\left(X \backslash x, S^{m} \Omega_{X}^{1}\right)$ and $\left[H^{0}\left(X^{\prime} \backslash x^{\prime}, S^{m} \Omega_{X^{\prime}}^{1}\right)\right]^{\mathbb{Z}_{2}}$. The following then holds:

$\operatorname{dim}\left[H^{0}\left(Y \backslash E, S^{m} \Omega_{Y}^{1}\right) / H^{0}\left(Y, S^{m} \Omega_{Y}^{1}\right)\right]=\operatorname{dim}\left[f^{*}\left[H^{0}\left(X^{\prime} \backslash x^{\prime}, S^{m} \Omega_{X^{\prime}}^{1}\right)\right]^{\mathbb{Z}_{2}} / g^{*} H^{0}\left(Y, S^{m} \Omega_{Y}^{1}\right)\right.$

Below we give a coordinate chart approach to find this dimension. 
Consider the blow up map $f:\left(\mathbb{C}^{2}, u, v\right) \rightarrow\left(\mathbb{C}^{2}, z_{1}, z_{2}\right)$ given by $(u, v) \rightarrow(u, u v)$. Consider also the double ramified covering $g:\left(\mathbb{C}^{2}, u, v\right) \rightarrow\left(\mathbb{C}^{2}, x, y\right)$ given by $(u, v) \rightarrow$ $\left(u^{2}, v\right)$.

The relations between the differentials in the different coordinate charts $\mathbb{C}^{2}$ are:

$$
f^{*} d z_{1}=d u \quad f^{*} d z_{2}=u d v+v d u
$$

and

$$
g^{*} d x=2 u d u \quad g^{*} d y=d v
$$

We may write the pullback by $f$ of a symmetric differential monomial on $\left(\mathbb{C}^{2}, z_{1}, z_{2}\right)$ as:

$$
f^{*}\left(d z_{1}^{m_{1}} d z_{2}^{m_{2}}\right)=\sum_{i=0}^{m_{2}}\left(\begin{array}{c}
m_{2} \\
i
\end{array}\right) u^{m_{2}-i} v^{i} d v^{m_{2}-i} d u^{m_{1}+i}
$$

So in any such pullback of order $m\left(m_{1}+m_{2}=m\right)$ there is a term in $d u^{m}$ with no power of $u$ in the coefficient (and all other terms have $d u$ with an order smaller than $m$ ). On the other hand, by (2.13) a symmetric differential $\omega=u^{i_{1}} v^{i_{2}} d u^{m_{1}} d v^{m_{2}}$ is a pullback by $g$ only if $i_{1} \geq m_{1}$. This and (2.13) imply that $f^{*}\left(h\left(z_{1}, z_{2}\right) d z_{1}^{m_{1}} d z_{2}^{m_{2}}\right)$ with $m_{1}+m_{2}=m$ is also a pullback by $g$ only if the Taylor expansion of $h$ has all terms with combined order in $z_{1}$ and $z_{2}$ to be greater or equal to $m$ (hence all terms of $f^{*}\left(h\left(z_{1}, z_{2}\right) d z_{1}^{m_{1}} d z_{2}^{m_{2}}\right)$ will have the coefficients with $u$ of order greater or equal to $m$ ).

Now there are $m+1$ symmetric differential monomials of order $m$ in $z_{1}$ and $z_{2}$. The number of all monomials in $z_{1}$ and $z_{2}$ of degree less than $m$ is $1+2+\ldots+m$. Hence the number symmetric differentials of the form $\omega=z_{1}^{i_{1}} z_{2}^{i_{2}} d z_{1}^{m_{1}} d z_{2}^{m_{2}}$ having $m_{1}+m_{2}=m$ such that $f^{*} \omega$ is not a pullback by $g$, i.e. $i_{1}+i_{2}<m$, is $\frac{1}{2} m^{3} \bmod m^{2}$. Recall that the goal is to find $\operatorname{dim}\left[f^{*}\left[H^{0}\left(X^{\prime} \backslash x^{\prime}, S^{m} \Omega_{X^{\prime}}^{1}\right)\right]^{\mathbb{Z}_{2}} / g^{*} H^{0}\left(Y, S^{m} \Omega_{Y}^{1}\right)\right.$. Hence we should only count the symmetric differentials that are $\mathbb{Z}_{2}$ invariant, i.e. $i_{1}+i_{2}=m \bmod 2$, which gives $\frac{1}{4} m^{3} \bmod m^{2}$. From $\operatorname{dim}\left[H^{0}\left(Y \backslash E, S^{m} \Omega_{Y}^{1}\right) / H^{0}\left(Y, S^{m} \Omega_{Y}^{1}\right)\right]=\operatorname{dim}\left[f^{*}\left[H^{0}\left(X^{\prime} \backslash\right.\right.\right.$ $\left.\left.x^{\prime}, S^{m} \Omega_{X^{\prime}}^{1}\right)\right]^{\mathbb{Z}_{2}} / g^{*} H^{0}\left(Y, S^{m} \Omega_{Y}^{1}\right)=\frac{1}{4} m^{3} \bmod m^{2}$, lemma 2.1 and formula (1.3) it follows $h^{1}\left(Y, S^{m} \Omega_{Y}^{1}\right)<C m^{2}$.

Proposition 2.3. Let $X$ be a surface with nodal singularities whose resolution $Y$ is a surface of general type. Then for $m>2$ :

$$
h^{2}\left(X, \hat{S}^{m} \Omega_{X}^{1}\right)=0
$$

Proof. From Serre duality for reflexive sheaves on normal surfaces, one obtains: 


$$
h^{2}\left(X, \hat{S}^{m} \Omega_{X}^{1}\right)=h^{0}\left(X,\left(\left(\hat{S}^{m} \Omega_{X}^{1}\right)^{\vee} \otimes \omega_{X}\right)^{\vee \vee}\right)
$$

The normality property of the reflexive sheaf $\left(\left(\hat{S}^{m} \Omega_{X}^{1}\right)^{\vee} \otimes \omega_{X}\right)^{\vee \vee}$ plus the reflexive nature of $\left(\hat{S}^{m} \Omega_{X}^{1}\right)^{\vee} \otimes \omega_{X}$ on $X_{\text {reg }}$ give:

$$
h^{0}\left(X,\left(\left(\hat{S}^{m} \Omega_{X}^{1}\right)^{\vee} \otimes \omega_{X}\right)^{\vee \vee}\right)=h^{0}\left(X_{r e g},\left(S^{m} \Omega_{X}^{1}\right)^{\vee} \otimes \omega_{X}\right)
$$

From the isomorphism $\Omega_{X}^{1}{ }^{\vee} \simeq \Omega_{X}^{1} \otimes \omega_{X}$ on $X_{\text {reg }}$, one derives the following equality:

$$
h^{2}\left(X, \hat{S}^{m} \Omega_{X}^{1}\right)=h^{0}\left(X_{r e g}, S^{m} \Omega_{X}^{1} \otimes \omega_{X}^{(1-m)}\right)
$$

Consider the the auxiliary vector bundles $S^{2 l} \Omega_{X}^{1} \otimes \omega_{X}^{-l}$ on $X_{\text {reg }}$. Suppose there is an $m>2$ such that $h^{2}\left(X, \hat{S}^{m} \Omega_{X}^{1}\right)>0$ then $h^{0}\left(X_{\text {reg }}, S^{m} \Omega_{X}^{1} \otimes \omega_{X}^{(1-m)}\right)>0$ by (2.15). Let $l=$ $q m, s \in H^{0}\left(X_{\text {reg }}, S^{m} \Omega_{X}^{1} \otimes \omega_{X}^{(1-m)}\right)$ be a nontrivial section and $w \in H^{0}\left(X_{\text {reg }}, \omega_{X}^{(m-2) q}\right)$ then $s^{2 q} \otimes w \in H^{0}\left(X_{r e g}, S^{2 l} \Omega_{X}^{1} \otimes \omega_{X}^{-l}\right)$. Since $m>2$ this implies that if $q \gg 0$ then $S^{2 l} \Omega_{X}^{1} \otimes \omega_{X}^{-l}$ has a nontrivial section vanishing at some point of $X_{\text {reg }}$.

The existence of a nontrivial section of $S^{2 l} \Omega_{X}^{1} \otimes \omega_{X}^{-l}$ vanishing at some point of $X_{\text {reg }}$ implies unstability properties of $\Omega_{X}^{1}$ on $X_{\text {reg }}$. Applying the stability theory of Bogomolov (and Mumford), it follows that there is a line bundle $L \subset \Omega_{X}^{1}$ on $X_{\text {reg }}$ such that the line bundle $\left(L^{2} \otimes \omega_{X}^{-1}\right)^{k}$ has nontrivial sections for some $k$ ([Bo78] or [De79]). The bigness of $K_{X}$ on $X_{r e g}$ finally implies that:

$$
h^{0}\left(X_{r e g}, L^{k}\right)=C k^{2}+O(k)
$$

Before proceeding we need the following lemma:

Lemma 2.4. Let $X$ be a projective surface with nodal singularities. Then there is a smoothing cover of $X$. More precisely, there is smooth projective surface $S$ with a finite map $f: S \rightarrow X$. In particular, the pre-image of the singular points consists of a finite set of smooth points.

Proof. The proof follows from the construction in theorem 2.5 of [KaBo98]. More precisely, for any surface $X$ with a finite number of quotient singularities $\left\{x_{1}, \ldots, x_{l}\right\}$ there is a projective variety $W$ with a holomorphic surjection $\pi: W \rightarrow X$. The fibration given by $\pi$ is a $\mathbb{P}^{n}$-bundle over $X \backslash \operatorname{Sing}(X)$ and has fibers $\mathbb{P}^{n} / G_{x_{i}}$ over the quotient singularity $x_{i}$. The group $G_{x_{i}}$ is the local fundamental group at the $x_{i}, G_{x_{i}}$ acts on $\mathbb{P}^{n} \times U$, where $U$ is a neighborhood of the $x_{i}$ in $X$. The action of the $G_{x_{i}}$ on $\mathbb{P}^{n}$ is faithful and projective and the action on $U$ is the action producing the quotient singularity $x_{i}$. The variety $W$ has singularities in codimension $\geq 3$, hence the intersection of a general codimension $n$ plane with $W$ is our smooth $S$ and $f=\left.\pi\right|_{S}$. 
By the previous lemma, there is a smoothing cover of $X, f: S \rightarrow X$. Denote by $T$ the pre-image on $S$ of the set of nodal points of $X$. Let $d f: f^{*} \Omega_{X}^{1} \rightarrow \Omega_{S}^{1}$ be the differential map. Consider the rank one subsheaf of $\left.\Omega_{S}^{1}\right|_{S \backslash T}$ on $S \backslash T$ which is the image sheaf $\mathcal{S}=d f(L)$. Since $T$ has codimension 2 on $S$, one can extend $\mathcal{S}$ to rank one coherent subsheaf on $S$ of $\Omega_{S}^{1}$. Let $L^{\prime} \subset \Omega_{S}^{1}$ be the line bundle which is the saturation of $\mathcal{S}$ on $\Omega_{S}^{1}$. The growth of the number of sections of $L^{k}$ described in (2.16) implies that:

$$
h^{0}\left(S, L^{\prime k}\right) \geq C k^{2}+O(k)
$$

On the other hand one has the general result stating that, on any complex surface $Y$, if $F \subset \Omega_{Y}^{1}$ is a line bundle, then growth of $h^{0}\left(S, F^{k}\right)$ is at most linear on $k$, which gives the desired contradiction.

So far the results we have gathered can be used to show the existence of symmetric differentials, i.e. sections of $H^{0}\left(X, \hat{S}^{m} \Omega_{X}^{1}\right)$ ), on nodal hypersurfaces $X \subset \mathbb{P}^{3}$ with enough nodes.

Corollary 2.5. Let $X$ be a nodal hypersurface of degree $d$ and $l$ nodes in $\mathbb{P}^{3}$. If $l>$ $\frac{8}{3}\left(d^{2}-\frac{5}{2} d\right)$ then :

$$
h^{0}\left(X, \hat{S}^{m} \Omega_{X}^{1}\right) \geq\left[\frac{l}{4}+\frac{1}{6}\left(10 d-4 d^{2}\right)\right] m^{3}+O\left(m^{2}\right)
$$

Proof. Let $Y$ be the minimal resolution of $X$ and $\left\{x_{i}\right\}_{i=1, \ldots, l}$ be the 1 nodes of $X$. The Riemann-Roch formula (2.6), the formula (2.4') and the leading term of the local Euler Characteristics $\chi\left(Y, S^{m} \Omega_{Y}^{1}\right)_{x_{i}}$ found in lemma 2.1 give:

$$
\chi\left(X, \hat{S}^{m} \Omega_{X}^{1}\right)=\left[\frac{1}{6}\left(10 d-4 d^{2}\right)+\frac{l}{4}\right] m^{3}+O\left(m^{2}\right)
$$

Hence if $l>\frac{8}{3}\left(d^{2}-\frac{5}{2} d\right)$ the Euler characteristic $\chi\left(X, \hat{S}^{m} \Omega_{X}^{1}\right)>0$ and grows with $m$ as $m^{3}$. The result follows since $h^{2}\left(X, \hat{S}^{m} \Omega_{X}^{1}\right)=0$ by proposition 2.3 .

The goal of this section is not yet achieved. The sections of $H^{0}\left(X, \hat{S}^{m} \Omega_{X}^{1}\right)$ do not give, apriori, symmetric differentials on the resolution $Y$. They correspond to sections of $H^{0}\left(Y \backslash E, S^{m} \Omega_{Y}^{1}\right)$. We would like to point out, that we have immediately a bit more. It follows from a result of Miyaoka ([Mi83], see also corollary 4.7 of [Wa93]) that for quotient singularities:

$$
h^{0}\left(Y \backslash E, S^{m} \Omega_{Y}^{1}\right)=h^{0}\left(Y, S^{m} \Omega_{Y}^{1}(\log E)\right)
$$

Therefore if $X$ has enough nodes then $Y$ has symmetric log-differentials. In the next result, we go farther and show the existence of the desired symmetric differentials on $Y$. 
Theorem 2.6. Let $X$ be a nodal hypersurface of degree $d$ and $l$ nodes in $\mathbb{P}^{3}$ and $Y$ its minimal resolution. If $l>\frac{8}{3}\left(d^{2}-\frac{5}{2} d\right)$, then $Y$ has symmetric differentials. More precisely:

$$
h^{0}\left(Y, S^{m} \Omega_{Y}^{1}\right) \geq\left[\frac{l}{4}+\frac{1}{6}\left(10 d-4 d^{2}\right)\right] m^{3}+O\left(m^{2}\right)
$$

There are nodal hypersurfaces of degree $d \geq 6$ with $l>\frac{8}{3}\left(d^{2}-\frac{5}{2} d\right)$ nodes.

Proof. Consider the exact sequence :

$$
0 \rightarrow S^{m} \Omega_{Y}^{1} \rightarrow S^{m} \Omega_{Y}^{1}(\log E) \rightarrow \mathcal{Q} \rightarrow 0
$$

where the sheaf $\mathcal{Q}=S^{m} \Omega_{Y}^{1}(\log E) / S^{m} \Omega_{Y}^{1}$ is supported on the exceptional locus $E$.

To start with, we want to find the asymptotic growth of $h^{0}(Y, \mathcal{Q})$. Let $\left(U_{i}, E_{i}\right)$ be the germ of a neighborhood of an exceptional curve $E_{i}$. The long cohomology sequence associated with (2.19) on $U_{i}$ and $h^{1}\left(U_{i}, S^{m} \Omega_{Y}^{1}\right)=O\left(m^{2}\right),(2.12)$, give that $h^{0}\left(U_{i}, \mathcal{Q}\right)=$ $\operatorname{dim}\left(H^{0}\left(U_{i}, S^{m} \Omega_{Y}^{1}\left(\log E_{i}\right)\right) / H^{0}\left(U_{i}, S^{m} \Omega_{Y}^{1}\right)\right)+O\left(m^{2}\right)$.

To proceed we recall a result of Miyaoka (see corollary 4.7 of [Wa93] or [Mi83]) stating that for germs $(U, E)$ of resolutions of quotient singularities:

$$
H^{0}\left(U \backslash E, S^{m} \Omega_{U}^{1}\right)=H^{0}\left(U, S^{m} \Omega_{U}^{1}(\log E)\right)
$$

Combining all the above with (1.3) and lemma 2.2, it follows that:

$$
h^{0}\left(E_{i}, \mathcal{Q}\right)=\frac{1}{4} m^{3}+O\left(m^{2}\right)
$$

and

$$
h^{0}(Y, \mathcal{Q})=\frac{l}{4} m^{3}+O\left(m^{2}\right)
$$

Next, we want to find a lower bound for $h^{1}\left(Y, S^{m} \Omega_{Y}^{1}\right)$. Consider the exact sequence (2.19) tensored with the line bundle $\mathcal{O}\left((1-m) K_{Y}\right)$ and use the isomorphism $\left.\mathcal{O}\left(K_{Y}\right)\right|_{U_{i}} \simeq$ $\mathcal{O}_{U_{i}}$ for the neighborhood germ $\left(U_{i}, E_{i}\right)$ of a $(-2)$-curve to obtain:

$$
0 \rightarrow S^{m} \Omega_{Y}^{1} \otimes \mathcal{O}\left((1-m) K_{Y}\right) \rightarrow S^{m} \Omega_{Y}^{1}(\log E) \otimes \mathcal{O}\left((1-m) K_{Y}\right) \rightarrow \mathcal{Q} \rightarrow 0
$$

The associated long cohomology sequence gives:

$$
H^{0}\left(Y, S^{m} \Omega_{Y}^{1}(\log E) \otimes \mathcal{O}\left((1-m) K_{Y}\right)\right) \rightarrow H^{0}(Y, \mathcal{Q}) \rightarrow H^{1}\left(Y, S^{m} \Omega_{Y}^{1} \otimes \mathcal{O}\left((1-m) K_{Y}\right)\right)
$$

Serre duality gives $h^{1}\left(Y, S^{m} \Omega_{Y}^{1}\right)=h^{1}\left(Y, S^{m} \Omega_{Y}^{1} \otimes \mathcal{O}\left((1-m) K_{Y}\right)\right)$. Hence if we show that $h^{0}\left(Y, S^{m} \Omega_{Y}^{1}(\log E) \otimes \mathcal{O}\left((1-m) K_{Y}\right)\right)=0$, it follows that: 


$$
h^{1}\left(Y, S^{m} \Omega_{Y}^{1}\right) \geq \frac{l}{4} m^{3}+O\left(m^{2}\right)
$$

The inequality (2.18) then follows from proposition 2.3 and the Euler characteristic $\left.\chi\left(Y, S^{m} \Omega_{Y}^{1}\right)=\frac{1}{6}\left(10 d-4 d^{2}\right)\right] m^{3}+O\left(m^{2}\right)$ being constant along a flat family.

The equality (2.20) implies that $H^{0}\left(Y, S^{m} \Omega_{Y}^{1}(\log E) \otimes \mathcal{O}\left((1-m) K_{Y}\right)\right)=H^{0}(Y \backslash$ $\left.E, S^{m} \Omega_{Y}^{1} \otimes \mathcal{O}\left((1-m) K_{Y}\right)\right)$. On the other hand, it follows from proposition 2.3 and its proof that $h^{0}\left(Y \backslash E, S^{m} \Omega_{Y}^{1} \otimes \mathcal{O}\left((1-m) K_{Y}\right)\right)=h^{0}\left(X_{\text {reg }}, S^{m} \Omega_{X}^{1} \otimes \mathcal{O}\left((1-m) K_{X}\right)\right)=$ $h^{2}\left(X, \hat{S}^{m} \Omega_{X}^{1}\right)=0$.

Segre showed in [Se47] that the maximum number of nodes $l(d)$ allowed in an hypersurface of degree $d$ satisfies $l(d) \geq \frac{1}{4} d^{2}(d-1)$. The lower bound for $l(d)$ of Segre gives that for $d \geq 9$ then there are nodal hypersurfaces with $l>\frac{8}{3}\left(d^{2}-\frac{5}{2} d\right)$ nodes. The cases $d=6,7,8$ follow from other specific lower bounds already found, see table in [Mi83]. For the case $d=5$ there are not enough nodes, the maximum number of nodes is 31 [Be83] and we needed 33 .

Remark: As was mentioned in the introduction of this section, the theorem 2.6 gives us an example of the jumping of the number of symmetric differentials on a smooth family of projective manifolds. This example, a family of smooth hypersurfaces $X_{t}$ of degree $d \geq 6$ in $\mathbb{P}^{3}$ specializing to the resolution $Y$ of a nodal hypersurface with enough nodes, is perhaps the most striking in its consequences and simplicity.

\section{HyPERBOLICITY OF NODAL HYPERSURFACES}

We prove in this section that the nodal hypersurfaces $X \subset \mathbb{P}^{3}$ whose number of nodes are sufficiently large when compared with their degrees are algebraically quasi-hyperbolic. The main tools are the existence of symmetric differentials proved in section 2 and the restrictions that they impose on curves with a fixed geometric genus in $X$.

A nontrivial symmetric differential $\omega \in H^{0}\left(Y, S^{m} \Omega_{Y}^{1}\right)$ defines a multi-foliation on $Y$. Recall that there is a natural bijection between $H^{0}\left(Y, S^{m} \Omega_{Y}^{1}\right)$ and $H^{0}\left(\mathbb{P}\left(\Omega_{Y}^{1}\right), \mathcal{O}(m)\right)$. If $\omega \in H^{0}\left(Y, S^{m} \Omega_{Y}^{1}\right)$ and $y \in Y$ then $\omega(y)$ defines naturally a polynomial of order $\mathrm{m}$ on $\mathbb{P}\left(\Omega_{Y}^{1}\right)_{y} \simeq \mathbb{P}^{1}$. The zeroes of $\omega(y)$ in $\mathbb{P}\left(\Omega_{Y}^{1}\right)_{y}$ determine the directions that the local leaves, of the multi-foliation defined by $\omega$, can take at $y$. Let $C$ be a smooth curve and $f: C \rightarrow Y$ be holomorphic map which is bimeromorphic onto its image. The curve $f(C)$ is a leaf of the multi-foliation defined by $\omega$ if the differential pullback $f^{*} \omega \in H^{0}\left(C,\left(\Omega_{C}^{1}\right)^{\otimes m}\right)$ is trivial. There is another way to describe the condition of being a leaf of $\omega$. Consider the lift of the map $f: C \rightarrow Y$ to a map $t_{f}: C \rightarrow \mathbb{P}\left(\Omega_{Y}^{1}\right)$. 
The curve $f(C)$ is a leaf if $t_{f}(C) \subset\left(s_{\omega}\right)_{0}$, where $\left(s_{\omega}\right)_{0}$ is the zero locus of the section $s_{\omega} \in H^{0}\left(\mathbb{P}\left(\Omega_{Y}^{1}\right), \mathcal{O}(m)\right)$ corresponding to $\omega$.

Theorem 3.1. Let $X$ be a nodal hypersurface of $\mathbb{P}^{3}$ with $l>\frac{8}{3}\left(d^{2}-\frac{5}{2} d\right)$ nodes, then $X$ is algebraically quasi-hyperbolic.

Proof. The result follows if we show that the minimal resolution $Y$ of $X$ is algebraically quasi-hyperbolic. Let $\phi_{m}: \mathbb{P}\left(\Omega_{Y}^{1}\right) \rightarrow \mathbb{P}^{l}$ be the rational map defined by the linear system $|\mathcal{O}(m)|$. Denote by $Z_{m} \subset \mathbb{P}\left(\Omega_{Y}^{1}\right)$ the locus consisting of the union of the positive dimensional fibers of $\phi$ and the base locus $B_{m}$ of $|\mathcal{O}(m)|$. The condition on the number of nodes implies, by the theorem 2.6, that $H^{0}\left(Y, S^{m} \Omega_{Y}^{1}\right) \uparrow m^{3}$. Hence the locus $Z_{m}$ consists of a finite union of irreducible components. Let $C$ be a smooth curve and $f: C \rightarrow Y$ be holomorphic map which is bimeromorphic onto its image. The curve $f(C)$ is said to be irregular if its lift $t_{f}(C) \subset Z_{m}$, otherwise it is called regular. The set of irregular curves can be broken into two groups: the curves that are leaves of all multi-foliations defined by the elements of $H^{0}\left(Y, S^{m} \Omega_{Y}^{1}\right)$ (i.e. the ones whose lift lies on the base locus $\left.B_{m}\right)$; the curves whose lift lie on the positive dimensional fibers of $\phi$.

Let $C^{\prime}$ be a regular curve with normalization $f: C \rightarrow C^{\prime} \subset Y$. The regularity of $C^{\prime}$ implies that there is a symmetric differential $\omega \in H^{0}\left(Y, S^{m} \Omega_{Y}^{1}\right)$ such that the differential pullback $f^{*} \omega \in H^{0}\left(C,\left(\Omega_{C}^{1}\right)^{\otimes m}\right)$ is nontrivial but vanishes somewhere. Hence $\operatorname{deg}_{C}\left(\Omega_{C}^{1}\right)^{\otimes m}>0$ and $C$ can not be rational or elliptic.

If $C^{\prime}$ is irregular then its lift lies on $Z_{m}$. The number of curves that lift into irreducible components of $Z_{m}$ that are not horizontal and 2-dimensional is clearly finite. The finiteness of the number of curves that lift to the 2-dimensional horizontal components of $Z_{m}$ is more delicate. These components have a naturally defined foliation on them which permits the use of the theory of foliations. In fact, the theory of algebraic foliations and $Y$ being of general type give that there are at most finitely many rational and elliptic curves lifting to any of the 2-dimensional horizontal components of $Z_{m}$, which finishes the proof (for more details see [Bo77] and [Jo78] and also [De79]).

\section{REFERENCES}

[At58] M.Atiyha, On analytic surfaces with double points, Proc. Roy. Soc. London. Ser. A 247 (1958), 237-244.

[Be79] A.Beauville, Sur le nombre de points doubles d'une surface dans $\mathbb{P}^{3}(\mu(5)=31)$, Journees de Geometrie Algebriquue d'Angers, Juillet 1979, Sijthoff \& Noordhoof, Alphen ann den Rijn, Germantown, Md 247 (1980), 207-215.

[B196] R.Blache, Chern classes and Hirzebruch-Riemann-Roch theorem for coherent sheaves on complex projective orbifolds with isolated singularities, Math. Z. 222 (1996), 7-57.

[Bo77] F.Bogomolov, Families of curves on a surface of general type, Soviet Math. Dokl. 18 (1977), 1294-1927.

[Bo78] F.Bogomolov, Holomorphic tensors and vector bundles, Izvestya A.N. USSR 42 (6) (1978), 1227 - 1287 (English translation Math USSR Izvestya 1979 v 13 (1) p 499 -544).

[Br71] P.Bruckmann, Tensor differential forms on algebraic varieties, Izvestya A.N. USSR 35 (1971), (English translation Math USSR Izvestya 1979 v 13 (1) p 499 -544).

[Cl86] H.Clemens, Curves on generic hypersurfaces, Ann. Sci. ENS 19 (1986), 629-636.

[De79] M.Deschamps, Courbes de genre geometrique borne sur une surface de type general (d'apres F. Bogomolov), Lect. Notes in Math. (Springer Verlag) 710 (1979), 233-247. 
[DeEl00] P. Demailly; J. El Goul, Hyperbolicity of generic surfaces of high degree in projective 3-space, Amer. J. Math. 122 (2000), 515-546.

[Ei88] L.Ein, Subvarieties of generic complete intersections, Inv. Math. 94 (1988), 163-169.

[El03] El Goul, Logarithmic jets and hyperbolicity, Osaka J. Math. 40 (2003), 469-491.

[GrGr79] M. Green; P.Griffiths, Two applications of algebraic geometry to entire holomorphic maps 3-space, The Chern Symposium 1979 (Springer Verlag) (1980), 41-74.

[Jo78] J-P, Jouanolou, Hypersurfaces solutions d'une equation de Pfaff analytique, Math. Annalen 232 (1978), 239-248.

[KaBo98] L.Katzarkov; F.Bogomolov, Complex projective surfaces and infinite groups, G.A.F.A. 8 (1998), 243-272.

[La86] S.Lang, Hyperbolic and Diophantine analysis, Bull. Amer. Math. Soc. Z. 14 (1986), 159-205.

[La00] A.Langer, Chern classes of reflexive sheaves on normal surfaces, Math. Z. 235 (2000), 591-614.

[Mc98] M.McQuillan, Diaphontine approximations and foliations, Publ. IHES 87 (1998), 121-174.

[Mi3] Y.Miyaoka, The maximal number of quotient singularities on surfaces with given numerical invariants, Math. Ann. 268 (1984), 159-171.

[Sa79] F.Sakai, Symmetric powers of the cotangent bundle and classification of algebraic varieties, Lect. Notes in Math. (Springer Verlag) 732 (1979), 545-563.

[Se47] B.Segre, Sul massimo numero di nodi delle superficie di dato ordine, Bull. U.M.I 2 (1947), 204-212.

[ShZa00] B. Shiffman; M. Zaidenberg, Two classes of hyperbolic surfaces in $\mathbb{P}^{3}$, Int. J. Math. 11 (2000), 65-101.

[SiYe96] Y-T.Siu; S.K.Yeung, Hyperbolicity of the complement of a generic smooth of high degree in the complex projectiveplane, Inv. Math. 124 (1996), 573-618.

[Vo96] C.Voisin, On a conjecture of Clemens on rational curves on hypersurfaces, J. Diff. Geom. 44 (1996), 200-213.

[Wa93] J. Wahl, Second Chern class and Riemann-Roch for vector bundles on resolutions of surface singularities., Math. Ann. 295 (1993), 81-110.

[Xu94] G.Xu, Subvarieties of general hypersurfaces in projective space, J. Diff. Geom. 39 (1994), 139-172.

Courant Institute for Mathematical Sciences, New York University, New York, Ny 10012

Department of Mathematics, University of Miami, Coral Gables, FL 33124

E-mail address: bogomolo@cims.nyu.edu, bdeolive@math.miami.edu 